\title{
Cambios Clínicos e Imagenológicos en Terapias no Invasivas en Articulaciones Temporomandibulares con Alteraciones Óseas Degenerativas
}

\author{
Clinical and Imaging Changes with Non-Invasive Therapies in \\ Temporomandibular Joints with Degenerative Joint Disease \\ Gustavo Moncada'; Constanza Valdés'; Montserrat Casals'; \\ Carlos Marholz²; Valeria Ramirez' ${ }^{1}$ \& Clemente Prieto'
}

MONCADA, G.; VALDÉS, C.; CASALS, C.; MARHOLZ, C.; RAMIREZ, V. \& PRIETO, C. Cambios clínicos e imagenológicos en terapias no invasivas en articulaciones temporomandibulares con alteraciones óseas degenerativas. Int. J. Odontostomat., 15(3):712-718, 2021.

RESUMEN: El objetivo del presente estudio fue describir los cambios clínicos e imagenológicos de las terapias no invasivas aplicadas a pacientes con alteraciones óseas degenerativas de las articulaciones temporomandibulares (ATM). Metodología: Se evaluaron 25 pacientes con alteraciones óseas degenerativas de las ATM, sin tratamiento previo de trastornos temporomandibulares (TTM) al momento del diagnóstico. Se realizó tratamiento no invasivo y un año después fueron evaluados según criterios clínicos e imagenológicos DC/TMD y Ahmad. Los resultados fueron presentados por medio de estadística descriptiva, odds ratio con sus respectivos intervalos de confianza, comparaciones de medianas y correlaciones. Se estudiaron 50 ATM, $72 \%$ mujeres (32,2 años promedio). Se observó mejora significativa en los parámetros: dolor $(p=0,0001)$, sinovitis $(p=0,001)$ e incremento de la esclerosis del trabeculado óseo $(p=0,051)$ a un año post-tratamiento. Después de un año del establecimiento de terapias no invasivas en pacientes con alteraciones óseas degenerativas de las ATM, se observaron cambios positivos tanto clínicos como imagenológicos, reduciéndose significativamente la sintomatología dolorosa, limitándose la progresión del daño óseo degenerativo, y observándose recuperación de los casos de sinovitis.

PALABRAS CLAVE: ATM, alteración ósea degenerativa, terapia no invasiva.

\section{INTRODUCCIÓN}

Los trastornos temporomandibulares (TTM) comprenden un grupo de condiciones músculoesqueletales y neuromusculares que incluyen las articulaciones temporomandibulares (ATM), los músculos masticatorios y/o estructuras asociadas y han sido identificados como la principal causa de dolor orofacial después del dolor dentario (Okeson, 2014). La etiología multifactorial de los TTM se relaciona con aspectos funcionales, estructurales y psicológicos del paciente, factores que pueden contribuir de manera aislada o combinados al diagnóstico, pronóstico y manejo terapéutico (Slade et al., 2013). La mayor prevalencia de TTM se observa en el segmento etario entre 18 y 45 años y es más frecuente en mujeres (Fillingim et al., 2011). La prevalencia de los TTM varía de manera importante en la literatura debido a diferencias en la terminología descriptiva, recolección de datos, análisis estadísticos y selección de la muestra (de Leeuw et al., 2018). La prevalencia de TTM en la población oscila entre $40 \%$ a $60 \%$ y se estima que entre un 3,6 $\%$ a $7 \%$ de los individuos con TTM requieren tratamiento (Okeson).

En las ATM, cuando los requerimientos superan las capacidades adaptativas, curativas y regenerativas de los tejidos, se inician deterioros estructurales como la alteración ósea degenerativa, caracterizada por la progresiva degradación del fibrocar-

\footnotetext{
${ }^{1}$ Universidad de Los Andes, Santiago, Chile.

${ }^{2}$ Universidad Andrés Bello, Santiago, Chile.
} 
tílago y remodelado o deterioro óseo subcondral, generalmente acompañado de dolor y limitación funcional, debido a la restringida capacidad de autoreparación del fibrocartílago (Roberts 2018).

Los estudios de tomografía computada y de resonancia nuclear magnética, se han establecido como gold standard para definir los cambios morfológicos de los tejidos duros y la determinación de la posición, configuración, forma del disco y otros tejidos blandos de la ATM. De esta manera, las imágenes de la ATM con alteraciones óseas degenerativas muestran alta evidencia de compromiso estructural, mayoritariamente localizado en el cóndilo mandibular, observándose baja correlación entre cambios condilares y dolor, reforzando la necesidad del complemento clínico e imagenológico en el diagnóstico de estas patologías (Palconet et al., 2012).

En cuanto al tratamiento de las alteraciones óseas degenerativas, el objetivo del tratamiento es reducir o eliminar la sintomatología dolorosa, restaurar la función mandibular, reducir la necesidad de futuros tratamientos, limitar la progresión del TTM, controlar el daño y restaurar un estilo vida "normal". El limitar la progresión de la enfermedad incluye evitar el incremento del trastorno degenerativo junto con intentar mantener la relación del complejo disco-condilar, los tejidos blandos articulares, la capacidad de lubricación articular y la capacidad de soportar cargas en el tiempo. La gama de tratamientos que permiten lograr estos propósitos se clasifican como terapias no invasivas, terapias mínimamente invasivas e invasivas (de Leeuw et al.).

Las revisiones sistemáticas y meta-análisis de diversas modalidades terapéuticas muestran que no existe evidencia que indique que las respuestas a las terapias no invasivas de los TTM son diferentes a las intervenciones más complejas e invasivas (AlBaghdadi et al., 2014), reportando que, en la mayoría de los casos, todos los síntomas clínicos de la alteraciones óseas degenerativas en la ATM, mejoran en un plazo de 30 días con tratamientos no invasivos. Sin embargo, el signo imagenológico de efusión articular, no presenta reducción total en ese plazo, comprobado con controles imagenológicos (Hosgor et al., 2017).

Actualmente, los tratamiento no invasivos parecen ser las medidas terapéuticas iniciales de elección y no presentan diferencias significativas con los resultados de los tratamientos quirúrgicos mínimamente invasivos (Al-Baghdadi et al.; de Leeuw et al.). A pesar de privilegiar los enfoques conservadores como primera línea terapéutica, y luego los mínimamente invasivos, estos se perciben como una herramienta terapéutica con mayor base fisiopatológica basada en la naturaleza inflamatoria crónica de las alteraciones óseas degenerativas (Manfredini et al., 2012). Los tratamientos no invasivos parecieran resolver en gran medida, los signos y síntomas clínicos de las alteraciones óseas degenerativas de la ATM en el corto plazo, siendo necesarias, en menor porcentaje, recurrir a medidas quirúrgicas mínimamente invasivas e invasivas (Hosgor et al.).

En este contexto, el objetivo del presente estudio fue describir los cambios clínicos e imagenológicos de las terapias no invasivas aplicadas a pacientes con alteraciones óseas degenerativas de las articulaciones temporomandibulares en un año plazo.

\section{MATERIAL Y MÉTODO}

Se llevó a cabo un estudio clínico, prospectivo no concurrente, observacional de una cohorte de pacientes, aprobado por el Comité de Ética Científica de la Universidad de Los Andes (CEC No 201951) y con respeto por los acuerdos de Helsinki. Se invitaron a participar a los pacientes que habían asistido a la consulta del Centro de Diagnóstico Integral (CODI), durante los años 2019-2020. Todos los pacientes invitados aceptaron y firmaron el consentimiento informado. La información de los pacientes en fichas e imágenes fue protegida codificándolas previamente.

Los criterios de Inclusión fueron: pacientes de ambos sexos, mayores de 18 años, primera consulta por TTM, diagnóstico clínico/imagenológico de alteraciones óseas degenerativas de al menos una ATM, tomografía computada y resonancia magnética al momento del diagnóstico y del control un año post tratamiento y aceptación del paciente del tratamiento no invasivo. Los criterios de exclusión fueron: pacientes con historia de trauma craneofacial o de ATM, antecedentes de alteraciones del metabolismo óseo, historia de intervenciones mínimamente invasivas de las ATM, malformaciones cráneo faciales, ingesta de corticoesteroides y antecedentes de artritis reumatoide.

El tamaño de muestra fue establecido de manera arbitraria, estableciéndose un total de 31 pacientes. Se recolectaron los antecedentes clínicos e imagenológicos de los pacientes y ATM en dos eta- 
pas, la basal, correspondiente a la primera consulta, al momento del diagnóstico clínico, y la del post tratamiento, un año después. La evaluación clínica y el tratamiento de los sujetos fue realizado por un operador calibrado según criterios DC-TMD (kappa de Cohen=0,81) (Schiffman et al., 2014) y la evaluación imagenológica fue realizada por un segundo operador entrenado y calibrado (kappa de Cohen $=0,92$ ) (Ahmad et al., 2009).

Se definió como outcome la intensidad del dolor reportada (escala de respuesta psicométrica visual análoga en escala 1-10). En los exámenes imagenológicos se evaluó la presencia de erosión de cortical ósea condilar, fosa o eminencia; alteraciones de forma condilar; aplanamiento condilar o de eminencia; pseudoquistes subcondrales; esclerosis del trabeculado condilar; esclerosis subcortical; efusión y posición discal a boca cerrada y abierta, según criterios Ahmad y DC-TMD (Ahmad et al.; Schiffman et al., 2014).

El protocolo terapéutico constó de una fase educativa, en la cual se explicó al paciente su patología, diagnóstico etiológico, incluyendo los factores de riesgo individuales de sus alteraciones óseas degenerativas, los objetivos terapéuticos, las fases del tratamiento y sus riesgos. Luego la fase de manejo conductual, que busca modificar malos hábitos o acciones consideradas factores de riesgo individual a través de la concientización, cambio de conducta y promoción de acciones protectoras y reparadoras articulares. Se indicó como esquema de complemento nutricional la ingesta del compuesto insaponificable de soya y palta (Piasclédine $®$, Laboratorios PHARMA INVESTI, Av. Andrés Bello 1495, Santiago, Chile) una vez al día, entre las comidas (con abundante agua) por un período de 3 meses (Catunda et al., 2016), vitamina D (400 mg/día) (Yilmaz et al., 2018) y vitamina C (1000 mg/día) (Chiu et al., 2016) de acuerdo con deficiencias individuales.La terapia física se indicó de acuerdo con los diagnósticos y sintomatología que padecía el paciente durante el tratamiento, considerando ejercicios de estiramiento, reposo, coordinación, fortalecimiento, resistencia, automasaje y revulsivos locales, con apoyo kinesiológico (Shimada et al., 2019). Todo lo anterior en paralelo con el uso de dispositivos ortopédicos intraorales de cobertura total, definidos como planos estabilizadores con guías de desoclusión anterior (Moraes et al., 2013; de Leeuw et al.). Todos los pacientes se mantuvieron en control durante un año.
Se realizó el análisis estadístico descriptivo y se evaluó la relación entre las variables considerando el anidamiento de las ATMs a nivel paciente, reportándose el odds ratio (OR), valor $p$, e intervalo de $95 \%$ de confianza (IC 95\%) (Stata 14.2).

\section{RESULTADOS}

De los 31 pacientes iniciales, 6 fueron excluidos, estudiándose 25 individuos (50 ATMs), participando 18 mujeres (72\%), entre 18 y 62 años y cuya edad media fue 32,2 años (desviación estándar [DE]: $11,4)$. No hubo pérdida de pacientes durante el período de estudio.

La intensidad del dolor orofacial en el examen inicial presentó una moda de 4 (EVA), y una mediana (p50) de 4 (rango intercuartílico [RIC]:3 - 6), que después de 1 año del tratamiento no invasivo, la moda se redujo a 0 y la mediana a 0 (RIC: 0 - 0). Los pacientes de sexo femenino presentaron rango mayor de intensidad de dolor, entre 0 a 9 (p50: 4; RIC: 3 - 7) y los hombres presentaron dolor de intensidad en el rango de 0 a 6 (p50: 4; RIC: 0 - 5). Luego de un año de tratamiento, 48 de las 50 ATMs estudiadas (96\%), presentó total eliminación de la sintomatología dolorosa $(E V A=0)$, con una mediana de 0.Esta disminución fue estadísticamente significativa (OR de regresión logística multinivel: 0,001; IC95\%: 0,0001 $-0,007 ; p$-value $=0,0001$ ). Solo una paciente presentó dolor un año después, con reducción de la intensidad de 7 a 3 en escala EVA. El análisis estadístico multinivel de las evaluaciones imagenológicos observadas al baseline comparadas al término del estudio, mostraron en general, estabilidad de los parámetros morfológicos de la ATM, observando aumento no significativo de la esclerosis del trabeculado óseo condilar $(p=0,051)$ y reducción significativa de las erosiones condilares $(p=0,009)(T a-$ bla I).

Se observó una importante recuperación de la salud articular en los casos de sinovitis, con reducción de 21 a 3 ATM con sinovitis $(p=0,001)$ y durante el período de observación, ninguna ATM presentó edema medular. El $98 \%(n=49)$ de las articulaciones presentaron desplazamientos discales y en el $96 \%(n=24)$ de los casos, ambas ATM estuvieron comprometidas. Los pacientes con alteraciones óseas degenerativas de las ATM, solicitaron atención básicamente por dolor. 
MONCADA, G.; VALDÉS, C.; CASALS, C.; MARHOLZ, C.; RAMIREZ, V. \& PRIETO, C. Cambios clínicos e imagenológicos en terapias no invasivas en articulaciones temporomandibulares con alteraciones óseas degenerativas. Int. J. Odontostomat., 15(3):712-718, 2021.

Tabla I. Cambios imagenológicos en las articulaciones temporomandibulares con alteraciones óseas degenerativas después de un año en individuos sometidos a tratamiento no invasivo.

\begin{tabular}{|c|c|c|c|c|c|c|c|c|}
\hline \multirow{2}{*}{$\begin{array}{c}\begin{array}{c}\text { Característica evaluada en } \\
\text { imágenes* }\end{array} \\
\end{array}$} & \multicolumn{2}{|c|}{ Basal } & \multicolumn{2}{|c|}{ Post-tratamiento } & \multirow[b]{2}{*}{ OR } & \multirow[b]{2}{*}{ valor $p$} & \multicolumn{2}{|c|}{$95 \%$ intervalo } \\
\hline & ATM & $\begin{array}{c}\text { Ambas } \\
\text { ATM }\end{array}$ & ATM & $\begin{array}{c}\text { Ambas } \\
\text { ATM }\end{array}$ & & & de cc & ianza \\
\hline Erosión cortical condilar & $\begin{array}{c}58 \% \\
(n=29)\end{array}$ & $\begin{array}{l}36 \% \\
(n=9)\end{array}$ & $\begin{array}{c}34 \% \\
(n=17)\end{array}$ & $\begin{array}{l}20 \% \\
(n=5)\end{array}$ & 0,26 & 0,009 & 0,09 & 0,71 \\
\hline Erosión fosa o eminencia & $\begin{array}{c}24 \% \\
(n=12)\end{array}$ & $\begin{array}{l}16 \% \\
(n=4)\end{array}$ & $\begin{array}{c}22 \% \\
(n=11)\end{array}$ & $\begin{array}{l}16 \% \\
(n=4)\end{array}$ & 0,84 & 0,767 & 0,26 & 2,69 \\
\hline Alteración forma condilar & $\begin{array}{c}88 \% \\
(n=44)\end{array}$ & $\begin{array}{l}76 \% \\
(n=19)\end{array}$ & $\begin{array}{c}80 \% \\
(n=40)\end{array}$ & $\begin{array}{c}68 \% \\
(n=17)\end{array}$ & 0,49 & 0,221 & 0,12 & 1,62 \\
\hline Aplanamiento apex Condilar & $\begin{array}{c}68 \% \\
(n=34)\end{array}$ & $\begin{array}{l}56 \% \\
(n=14)\end{array}$ & $\begin{array}{c}70 \% \\
(n=35)\end{array}$ & $\begin{array}{l}64 \% \\
(n=16)\end{array}$ & 1,29 & 0,721 & 0,32 & 5,28 \\
\hline $\begin{array}{l}\text { Aplanamiento eminencia } \\
\text { temporal }\end{array}$ & $\begin{array}{l}36 \% \\
(n=18)\end{array}$ & $\begin{array}{l}32 \% \\
(n=8)\end{array}$ & $\begin{array}{l}48 \% \\
(n=24)\end{array}$ & $\begin{array}{l}40 \% \\
(n=10)\end{array}$ & 3,41 & 0,073 & 0,89 & 13,02 \\
\hline Pseudoquiste subcondral & $\begin{array}{l}12 \% \\
(n=6)\end{array}$ & $\begin{array}{l}4 \% \\
(n=1)\end{array}$ & $\begin{array}{l}14 \% \\
(n=7)\end{array}$ & $\begin{array}{l}4 \% \\
(n=1)\end{array}$ & 1,27 & 0,729 & 0,32 & 5,00 \\
\hline Esclerosis trabeculado & $32 \%$ & $16 \%$ & $48 \%$ & $28 \%$ & & & & \\
\hline $\begin{array}{l}\text { subcortical } \\
\text { condilar }\end{array}$ & $(n=16)$ & $(n=4)$ & $(n=24)$ & $(n=7)$ & 2,89 & 0,051 & 0.99 & 8,42 \\
\hline
\end{tabular}

\section{DISCUSIÓN}

En el presente estudio, la notable disminución de la sinovitis evidenciada al control anual, demuestra el control inflamatorio intra-articular del tratamiento no invasivo y es interpretada como señal objetiva de éxito terapéutico a pesar que se debe profundizar el análisis de factores de comorbilidad que la perpetuaron en el $6 \%$ de las ATM. La sinovitis en la literatura se asocia a la liberación de MMP, VEGF y otras citoquinas, que explican la presencia de dolor, inflamación y destrucción del fibrocartílago y tejido óseo (Ernberg, 2017; Roberts et al., 2018).

El $24 \%$ de los pacientes $(n=6)$, no presentaron sintomatología dolorosa ni limitación funcional al baseline, sin embargo, sus imágenes mostraron erosión cortical condilar en el $50 \%$ ( $n=3$ de 6), efusión en los recesos articulares en el $50 \%$ y áreas pseudoquísticas condilares en el 33,3\% ( $n=2$ de 6). A pesar del bajo $\mathrm{n}$ de pacientes, estos resultados concuerdan con hallazgos previos de desplazamientos discales en pacientes asintomáticos en el $34 \%$ de voluntarios jóvenes que presentaron procesos de deterioro y abrasión del cartílago articular sin manifestaciones inflamatorias iniciales (Ribeiro et al., 1997).

En el presente estudio el $58 \%(n=29)$ de las ATM mostraron erosión de la cortical condilar al baseline, correspondiendo el 68,9 \% ( $n=20)$ a articulaciones femeninas $(36,37)$, coincidiendo con lo reportado previamente como hallazgo frecuente en pacientes con TTM, en el rango de prevalencia de $18 \%$ a 84
$\%$ (35). La explicación de la alta frecuencia femenina en edad reproductiva, se asocia con el rol de estrógenos y relaxina $\mathrm{H} 2$ en la predisposición a la inducción de la actividad enzimática de metaloproteinasas (MMP), interleuquinas (IL) y factor de necrosis tumoral (TNF), vinculado a la degradación del fibrocartílago (Hashem et al., 2006).

Los efectos de los tratamientos no invasivos (TNI) de los TTM en el tiempo (Abouelhuda et al., 2018), muestran que a medida que se ha incrementado la información sobre los resultados de las terapias mínimamente invasivas y quirúrgicas, se ha evidenciado menor invasividad, indicándose mayoritariamente terapias no invasivas para el inicio del tratamiento de alteraciones óseas degenerativas (Manfredini et al.; Freitas de Souza et al., 2012). El tratamiento no invasivo mostró significativa reducción del dolor en pacientes con alteraciones óseas degenerativas, (96\% de pacientes alcanzaron $E V A=0$ ), similar a lo observado previamente (de Leeuw et al.). En general la literatura señala que, en la mayoría de los casos, los TTM se estabilizan en el período de 6 a 12 meses después del inicio del tratamiento, logrando alivio sintomatológico con terapias no invasivas, en el rango de $50 \%$ a $94 \%$ de los casos (Randolph et al., 1990; Efeoglu et al., 2018). donde las condiciones no resueltas se asocian habitualmente a comorbilidades (Randhama et al., 2016).

Estos resultados refuerzan el hecho que las terapias no invasivas logran la resolución de las altera- 
ciones óseas degenerativas a través del manejo de los diferentes factores de riesgo individual, junto al fomento de la reparación, consiguiendo resultados evidenciables a largo plazo (Tanaka et al., 2004).

La literatura presenta múltiples modalidades de tratamientos no invasivos que aportan a su recuperación, incluyendo hábitos alimenticios (consistencia, tamaño); control de parafunciones y malos hábitos; medicamentos anti inflamatorios esteroidales o no, antidepresivos, relajantes musculares; recomendaciones para cambios en los estilos de vida; suplementos nutricionales; terapia física; uso de planos interoclusales y otros que logran manejar la sintomatología, a pesar que presentan efectos limitados para reestablecer el cartílago y el hueso dañado al interior de la ATM (Tanaka et al.; Randhawa et al., 2016).

Los dispositivos inter-oclusales son discutidos, a pesar de sus beneficios en la reducción significativa de la sintomatología dolorosa y mejoras funcionales. La literatura respalda su uso en pacientes con hábitos para-funcionales, sin embargo su rol aún se discute, a pesar de mitigar alteraciones dento-alveolares, sintomatología muscular, condición articular, cambios en la información propioceptiva y en distribución de los contactos oclusales y presentar efecto placebo (Jokubauskas et al., 2018), sin embargo, estas conclusiones se desprenden de estudios que difieren en diseños, protocolos diagnóstico y de tratamiento, generando resultados poco comparables respecto de su rendimiento clínico (Jokubauskas et al.). Revisiones sistemáticas concluyen favorablemente sobre la eficiencia del TNI en pacientes con bruxismo de sueño y alteraciones óseas degenerativas con planos, observándose que la eficiencia de los planos no presenta diferencias significativas con otras modalidades para el manejo del dolor (Jokubauskas et al.). Lo anterior demanda que la efectividad de los planos debe ser discutida en estudios clínicos controlados aleatorizados a largo plazo, que conduzca a revisiones sistemáticas y meta-análisis, para evaluar su efecto, incluyendo alteraciones óseas degenerativas de la ATM (Riley et al., 2020).

Al baseline, la asimetría de forma entre fosa glenoidea y cóndilo en las imágenes de tomografía computada, se observó de alta frecuencia, comprometiendo ambas ATM. Esta variación de la forma y reducción del tamaño condilar ha sido previamente reportada asociada al desplazamiento discal (Hasegawa et al., 2011).
Al baseline en este estudio, se observó alta frecuencia de aplanamiento de ápex condilar (68\%), y de estas el $56 \%$ fue bilateral, resultados que coinciden con lo observado previamente (Talaat et al., 2016), destacado como hallazgo común en pacientes con ADO. El cambio de forma de la eminencia del temporal expresado como aplanamiento fue de menor frecuencia (36\%) y de estos el $32 \%$ fue bilateral, hallazgos que ocurren como resultado del remodelado adaptativo o cambios óseos degenerativos secundario a desarreglos internos. Sin embargo, el aplanamiento óseo impresiona como interpretación subjetiva, si se compara con otros parámetros imagenológicos como erosión, sinovitis o cambios en la arquitectura del trabeculado y pareciera no ser una variable definitoria para el diagnóstico de la condición ósea (Ahmad et al.).

La presencia de áreas pseudoquísticas subcorticales, se observó al baseline en el $12 \%$ de los pacientes, sin diferencia por sexo (sólo uno presentó pseudoquistes en ambas ATM), frecuencia menor que la reportada previamente (40\%), a pesar que su estudio fue efectuado con imágenes de ortopantomografía y evaluó sólo la vertiente funcional anterior sin mostrar diferencia entre sexos (Zadik et al., 2015). En el presente estudio, después de 1 año de terapia no invasiva, se redujeron al $10 \%$ las áreas pseudoquísticas, presentando mejor rendimiento en los pseudoquísticas de menor tamaño, limitó su crecimiento y no se presentaron nuevas áreas pseudoquísticas al control anual.

La esclerosis condilar subcortical al baseline, se observó en el $32 \%$ de los casos, (16 \% bilateral), correspondiendo el $66 \%$ al sexo femenino, estos resultados son inferior al $62 \%$ mostrado en pacientes con artritis reumatoide (61), no incluidos en este estudio, mientras los presentes resultados son mayores al $20 \%$ publicado previamente en pacientes con alteraciones óseas degenerativas (Shahidi et al., 2018). Después de 1 año de terapia no invasiva, se incrementaron las áreas de esclerosis del trabeculado óseo condilar a $48 \%$, interpretado como respuesta mineralizadora reparativa frente a la lesión.

Otro cambio del trabeculado óseo fue la esclerosis subcortical de fosa/eminencia, encontrado al baseline en el $24 \%$ de los pacientes y de estas, el $75 \%$ correspondió a ATM de mujeres e importante frecuencia bilateral $(5,5 \%)$, frecuencia inferior al $48 \%$ previamente observada (Ottersen et al., 2019) y al 8,1 \% (Gil et al., 2012), estos cambios no fueron modificados por el tratamiento no invasivo después de un año (22 \%). 
En ninguna de las dos evaluaciones imagenológicas se observó esclerosis generalizada del trabeculado, presencia de osteofitos, cuerpo articular suelto, anquilosis ósea, hipoplasia o hiperplasia.

Los resultados del presente estudio permiten aceptar la hipótesis alternativa propuesta y muestran el rol protector clínico y morfológico del daño óseo degenerativo en las ATM, por medio de tratamiento no invasivo aplicados en pacientes con alteraciones óseas degenerativas, permitiendo a los pacientes la regresión a la media, ya que toda patología y en particular las enfermedades degenerativas tienden a pasar por ciclos, presentando periodos de reagudización. La explicación de este hecho podría relacionarse con la disminución de la sobrecarga articular en conjunto con los efectos de fármacos y dinámicas que promueven el remodelado óseo, permitiendo que se adapte a las necesidades fisiológicas del paciente, que se refleja especialmente en los resultados obtenidos en el incremento de la esclerosis subcortical condilar (Tanaka et al.; Akbulut et al., 2018), interpretado como respuesta mineralizadora reparativa.

La pérdida de la forma discal es una característica frecuente de los TTM. En esta muestra, el $84 \%$ de los discos presentaron deformación al baseline, caracterizada por el aplanamiento del margen posterior y/o plegamiento discal, mayoritariamente hacia anterior. Los desplazamientos discales y la sintomatología dolorosa no son indicadores que se asocien directamente con alteraciones óseas degenerativas, especialmente si se considera que las estructuras de la ATM pueden llegar a adaptarse muy bien y sin dolor a las diferentes posiciones del disco (Poluha et al., 2019), excepto cuando el disco se presenta desplazado sin reducción (Cortés et al., 2011).

Finalmente, los parámetros imagenológicos observados en el presente estudio fueron evaluados mediante escala categórica binarias, hecho que impide observar resultados en situaciones intermedias, sugiriéndose desarrollar clasificación de las variables de manera continua para lograr observaciones que permitan mejor discriminación de los niveles de severidad de las lesiones.

CONCLUSIONES. Las terapias no invasivas reducen significativamente la sintomatología dolorosa, presentando efecto protector y limitador de la progresión del daño óseo degenerativo en la ATM de pacientes con TTM y con alto rendimiento para la recuperación de la sinovitis.
MONCADA, G.; VALDÉS, C.; CASALS, C.; MARHOLZ, C.; RAMIREZ, V. \& PRIETO, C. Clinical and imaging changes with non-invasive therapies in temporomandibular joints with degenerative joint disease. Int. J. Odontostomat., 15(3):712718, 2021.

ABSTRACT: The objective of this study was to describe clinical and imaging changes of non-invasive therapies applied to patients with degenerative bone disorders of the temporomandibular joints (TMJ). To carry out this study, 25 patients with degenerative bone disorders of TMJ without previous treatment at the time of diagnosis, were evaluated. Non-invasive treatment was performed and one year later they were evaluated according to clinical and imaging criteria DC/TMD and Ahmad. Results were presented by descriptive statistics, odds ratio, confidence interval, comparisons of means, and correlations. 50 TMJs, $72 \%$ women, (32.2 years mean of age) were studied. Significant improvement was observed in the parameters: pain $(p=0.0001)$, synovitis $(p=0.001)$, and increased sclerosis of the bone trabeculae $(p=0,051)$ at one-year post-treatment. After one year of the establishment of non-invasive therapies in patients with degenerative joint disease of TMJ, it was observed positive changes, both clinical and imaging, reducing painful symptomatology, limiting effect on the progression of degenerative bone damage, and recovery of synovitis cases.

KEY WORDS: TMJ, degenerative bone disease, non-invasive therapy.

\section{REFERENCIAS BIBLIOGRÀFICAS}

Abouelhuda, A. M.; Khalifa, A. K.; Kim, Y. K. \& Hegazy, S. A. Noninvasive different modalities of treatment for temporomandibular disorders: review of literature. J. Korean Assoc. Oral Maxillofac. Surg., 44(2):43-51, 2018.

Ahmad, M.; Hollender, L.; Anderson, Q.; Kartha, K.; Ohrbach, R.; Truelove, E. L.; John, M. T. \& Schiffman, E. L. Research diagnostic criteria for temporomandibular disorders (RDC/TMD): development of image analysis criteria and examiner reliability for image analysis. Oral Surg. Oral Med. Oral Pathol. Oral Radiol. Endod., 107(6):844-60, 2009.

Akbulut, N.; Altan, A.; Akbulut, S. \& Atakan, C. Evaluation of the 3 $\mathrm{mm}$ thickness splint therapy on temporomandibular joint disorders (TMDs). Pain Res. Manag., 2018:3756587, 2018.

Al-Baghdadi, M.; Durham, J.; Araujo-Soares, V.; Robalino, S.; Errington, L. \& Steele, J. TMJ disc displacement without reduction management: a systematic review. J. Dent. Res., 93(7 Suppl.):37S-51S, 2014.

Catunda, I. S.; do E. Vasconcelos, B. C.; de S. Andrade, E. S. \& Costa, D. F. N. Clinical effects of an avocado-soybean unsaponifiable extract on arthralgia and osteoarthritis of the temporomandibular joint: preliminary study. Int. J. Oral Maxillofac. Surg., 45(8):1015-22, 2016.

Chiu, P. R.; Hu, Y. C.; Huang, T. C.; Hsieh, B. S.; Yeh, J. P.; Cheng, H. L.; Huang, L. W. \& Chang, K. L. Vitamin C protects chondrocytes against monosodium iodoacetate-induced osteoarthritis by multiple pathways. Int. J. Mol. Sci., 18(1):38, 2016. 
MONCADA, G.; VALDÉS, C.; CASALS, C.; MARHOLZ, C.; RAMIREZ, V. \& PRIETO, C. Cambios clínicos e imagenológicos en terapias no invasivas en articulaciones temporomandibulares con alteraciones óseas degenerativas. Int. J. Odontostomat., 15(3):712-718, 2021.

Cortés, D.; Exss, E.; Marholz, C.; Millas, R. \& Moncada, G. Association between disk position and degenerative bone changes of the temporomandibular joints: an imaging study in subjects with TMD. Cranio, 29(2):117-26, 2011.

de Leeuw, R.; Klasser, G. D. \& American Academy of Orofacial Pain. Orofacial Pain. Guidelines for Assessment, Diagnosis, and Management. 6a ed. Berlin, Quintessence, 2018.

Efeoglu, C.; Calis, A. S.; Koca, H. \& Yuksel, E. A stepped approach for the management of symptomatic internal derangement of the temporomandibular joint. J. Otolaryngol. Head Neck Surg., 47:33, 2018.

Ernberg, M. The role of molecular pain biomarkers in temporomandibular joint internal derangement. J. Oral Rehabil., 44(6):481-91, 2017.

Fillingim, R. B.; Ohrbach, R.; Greenspan, J. D.; Knott, C.; Dubner, R.; Bair, E.; Baraian, C.; Slade, G. D. \& Maixner, W. Potential psychosocial risk factors for chronic TMD: descriptive data and empirically identified domains from the OPPERA case-control study. J. Pain, 12:(11 Suppl.):T46-60, 2011.

Freitas de Souza, R.; Lovato da Silva, C. H.; Nasser, M.; Fedorowicz, Z. \& Al-Muharraqi, M. A. Interventions for the management of temporomandibular joint osteoarthritis. Cochrane Database Syst. Rev., 2012(4):CD007261, 2012.

Gil, C.; Santos, K. C. P.; Dutra, M. E. P.; Kodaira, S. K. \& Oliveira, J. $\mathrm{X} . \mathrm{MRI}$ analysis of the relationship between bone changes in the temporomandibular joint and articular disc position in symptomatic patients. Dentomaxillofac. Radiol., 41(5):367-72, 2012.

Hasegawa, H.; Saitoh, I.; Nakakura-Ohshima, K.; Shigeta, K.; Yoshihara, T.; Suenaga, S.; Inada, E.; Iwasaki, T.; Matsumoto, Y. \& Yamasaki, Y. Condylar shape in relation to anterior disk displacement in juvenile females. Cranio, 29(2):100-10, 2011.

Hashem, G.; Zhang, Q.; Hayami, T.; Chen, J.; Wang, W. \& Kapila, $\mathrm{S}$. Relaxin and beta-estradiol modulate targeted matrix degradation in specific synovial joint fibrocartilages: progesterone prevents matrix loss. Arthritis Res. Ther., 8(4):R98, 2006.

Hosgor, H.; Bas, B. \& Celenk, C. A comparison of the outcomes of four minimally invasive treatment methods for anterior disc displacement of the temporomandibular joint. Int. J. Oral Maxillofac. Surg., 46(11):1403-10, 2017.

Jokubauskas, L.; Baltrusaityte, A. \& Pileicikiene, G. Oral appliances for managing sleep bruxism in adults: a systematic review from 2007 to 2017. J. Oral Rehabil., 45(1):81-95, 2018.

Manfredini, D.; Rancitelli, D.; Ferronato, G. \& Guarda-Nardini, L. Arthrocentesis with or without additional drugs in temporomandibular joint inflammatory-degenerative disease: comparison of six treatment protocols. J. Oral Rehabil., 39(4):24551,2012

Moraes, A. R.; Sanches, M. L.; Ribeiro, E. C. \& Guimarães, A. S. Therapeutic exercises for the control of temporomandibular disorders. Dental Press J. Orthod., 18(5):134-9, 2013.

Okeson, J. P. Bell's Oral and Facial Pain. 7a ed. Chicago, Quintessence, 2014. pp.560.

Ottersen, M. K.; Abrahamsson, A. K.; Larheim, T. A. \& Arvidsson, L. Z. СBCT characteristics and interpretation challenges of temporomandibular joint osteoarthritis in a hand osteoarthritis cohort. Dentomaxillofac. Radiol., 48(4):20180245, 2019.

Palconet, G.; Ludlow, J. B.; Tyndall, D. A. \& Lim, P. F. Correlating cone beam CT results with temporomandibular joint pain of osteoarthritic origin. Dentomaxillofac. Radiol., 41(2):126-30, 2012.

Poluha, R. L.; De la Torre Canales, G.; Costa, Y. M.; Grossmann, E.; Bonjardim, L. R. \& Conti, P. C. R. Temporomandibular joint disc displacement with reduction: a review of mechanisms and clinical presentation. J. Appl. Oral Sci., 27:e20180433, 2019.

Randhawa, K.; Bohay, R.; Côté, P.; van der Velde, G.; Sutton, D.;
Wong, J. J.; Yu, H.; Southerst, D.; Varatharajan, S.; Mior, S.; et al. The effectiveness of noninvasive interventions for temporomandibular disorders: a systematic review by the Ontario Protocol for Traffic Injury Management (OPTIMa) Collaboration. Clin. J. Pain, 32(3):260-78, 2016.

Randolph, C. S.; Greene, C. S.; Moretti, R.; Forbes, D. \& Perry, H. T. Conservative management of temporomandibular disorders: a posttreatment comparison between patients from a university clinic and from private practice. Am. J. Orthod. Dentofacial Orthop., 98(1):77-82, 1990.

Ribeiro, R. F.; Tallents, R. H.; Katzberg, R. W.; Murphy, W. C.; Moss, M. E.; Magalhaes, A. C. \& Tavano, O. The prevalence of disc displacement in symptomatic and asymptomatic volunteers aged 6 to 25 years. J. Orofac. Pain, 11(1):37-47, 1997.

Riley, P.; Glenny, A. M.; Worthington, H. V.; Jacobsen, E.; Robertson, C.; Durham, J.; Davies, S.; Petersen, H. \& Boyers, D. Oralsplints for patients with temporomandibular disorders or bruxism: a systematic review and economic evaluation. Health Technol. Assess., 24(7):1-224, 2020.

Schiffman, E.; Ohrbach, R.; Truelove, E.; Look, J.; Anderson, G.; Goulet, J. P.; List, T.; Svensson, P.; Gonzalez, Y.; Lobbezoo, F.; et al. Diagnostic Criteria for Temporomandibular Disorders (DC/ TMD) for clinical and research applications: recommendations of the International RDC/TMD Consortium Network* and Orofacial Pain Special Interest Groupt. J. Oral Facial Pain Headache, 28(1):6-27, 2014.

Shahidi, S.; Salehi, P.; Abedi, P.; Dehbozorgi, M.; Hamedani, S. \& Berahman, N. Comparison of the bony changes of TMJ in patients with and without TMD complaints using CBCT. J. Dent. (Shiraz), 19(2):142-9, 2018.

Shimada, A.; Ishigaki, S.; Matsuka, Y.; Komiyama, O.; Torisu, T.; Oono, Y.; Sato, H.; Naganawa, T.; Mine, A.; Yamazaki, Y.; et al. Effects of exercise therapy on painful temporomandibular disorders. J. Oral Rehabil., 46(5):475-81, 2019.

Slade, G. D.; Bair, E.; Greenspan, J. D.; Dubner, R.; Fillingim, R. B.; Diatchenko, L.; Maixner, W.; Knott, C. \& Ohrbach, R. Signs and symptoms of first-onset TMD and sociodemographic predictors of its development: the OPPERA prospective cohort study. J. Pain, 14(12 Suppl.):T20-32.e1-3, 2013.

Talaat, W.; Al Bayatti, S. \& Al Kawas, S. CBCT analysis of bony changes associated with temporomandibular disorders. Cranio, 34(2):88-94, 2016.

Tanaka, E. E.; Arita, E. S. \& Shibayama, B. Occlusal stabilization appliance: evaluation of its efficacy in the treatment of temporomandibular disorders. J. Appl. Oral Sci., 12(3):238-43, 2004.

Yilmaz, A. D.; Yazicioglu, D.; Öncül, A. M. T.; Yilmaz, E. \& Eres, G. Vitamin $D$ receptor gene polymorphisms (Apa1 and Taq1) intemporomandibular joint internal derangement/osteoarthritis in a group of Turkish patients. Mol. Biol. Rep., 45(6):1839-48, 2018.

Zadik, Y.; Yitschaky, O.; Pikovsky, A.; Zini, A. \& Barenboim, S. F. Detection of mandibular condyle pesudocysts in panoramic radiographs: Digital and film-based radiology. Quintessence Int., 46(8):725-30, 2015.

Dirección para correspondencia:

Gustavo Moncada

Rehabilitación Oral

Facultad de Odontología

Universidad de Los Andes

Santiago - CHILE

E-mail: gmoncada@adsl.tie.cl 